\title{
Tensit - A simulation tool for nuclide transport, risk and dose calculations
}

\author{
J. J ones, U. Kautsky and C.F. Vahlund
}

SKB, Swedish Nuclear Fuel and Waste Management Co., 10240 Stockholm, Sweden

\begin{abstract}
The present paper presents, a probabilistic simulation package, Tensit, used for migration calculation of radionuclides from a deep repository for spent nuclear fuel to the biosphere. The package is based on pre-existing codes (Matlab, Simulink and the probabilistic engine @Risk) and is capable of performing migration calculations for the near field, far field and the biosphere for a high level waste repository. In addition to the compartment based models based on transfer factors, the code is also able to handle the more complex ecosystem models (based on flow of carbon and nutrients) either separately or in combination with the compartment models. Comparisons with other codes used for compartment based biosphere modelling as well as example of simple models, more advanced ecosystems and connected ecosystems Landscape model are shown in the paper.
\end{abstract}

\section{INTRODUCTION}

During the next period of years the Swedish Nuclear Fuel and Waste Management Co (SKB) performs site investigations for a future repository of spent nuclear fuel. The repository will be situated in crystalline rock at a depth of approximately $500 \mathrm{~m}$. The repository will consist of fuel contained in cast iron canisters with a shell of copper (used to provide the necessary corrosion protection). Also a number of safety assessments will be performed the near future in order to investigate the suitability of the system and of the studied sites.

Nuclide transport simulations (where the effects of a potential release of radionuclides are studied) are an essential part of a safety assessments performed to investigate the performance of the system. These calculations are (in the SKB case) based on different codes handling migration through the engineered system (the near field, which surrounds the copper canister around which the system is build) further through the geosphere (fractured crystalline rock in the Swedish concept) and finally to the biosphere where doses are calculated. In the present paper, the focus lies on biosphere migration and releases from the near-field. The geosphere will merely be regarded as an input function to the biosphere migration module.

There exist a large number of computer codes used for migration simulations in safety assessments of spent nuclear fuel repositories. These codes are generally well functioning and show corresponding results for the same problem (this has been shown in cross codes comparisons, for instance the PSACOIN [1]). However, many tools are specialised and may not easily be adapted to new simulation circumstances. An example of this is the inability to implement ecosystemmodels [2] in compartment based codes. It is however likely that many of the developers of the commercial codes would be able to implement such problems. This requires however that the models have been tested, validated and the different mechanisms considered are of importance. To do early investigations on various subjects including ecosystem modelling, it is of interest to have a software tool in which it is easy to investigate different mechanisms without having access to the source code. These commonly belong to the software developers and are normally not available for the users. 


\section{DEVELOPMENT OF THE CODE AND DEMONSTRATION OF ITS FUNCTIONALITY}

The migration simulation code presented in the current paper is written as an application in different commercial computer codes. The requirements on the chosen codes were that they should be widely spread (in the scientific community), user friendly and accurate.

For the migration calculations, Matlab and Simulink were the chosen codes [3]. These codes are suitable for solving problems in the field of numerical analysis, linear algebra and they also includes different graphical tools useful when presenting the results. For the current application when the capability to solve systems of differential equations is essential, Matlab is a suitable code since the solvers used are thoroughly tested, well documented, continuously developed and are possible to adapt for a large number of different problems (stiff and non stiff system of differential equations, adaptive time stepping necessary when performing simulation over a large time span etc.). The built in scripting language is very powerful, allowing for the user define problems in a very high level mathematical language.

Simulink is added to Matlab as a graphical user interface when solving differential equations. By using these two codes, a simulation package having a graphical interface with the capability of solving a large number of numerical problems is obtained. Both codes are available for a variety of computer platforms, hence the problem may be defined on a office PC and (of importance when simulating landscape models or for large probabilistic simulations) being processed on a high performance work station.

In order for the code to handle probabilistic simulations, the commercial code @Risk is used. This statistical software package works as a plug-in application for Microsoft Excel. Also this software is well spread and tested and provides a user friendly interface to assign probabilistic distributions to model parameters and to obtain statistics from probabilistic simulations. This program runs however not on the UNIX platform, input data for UNIX can however be generated on a standard PC and results from the UNIX simulation may be post processed on a PC.

The three different codes where combined into a package named Tensit. Tensit facilitates a software link between @Risk and Matlab/Simulink so that the two codes can interact. The user only specifies which Simulink model to simulate, the blocks that need sampled data and their distributions. General properties such as number of realisations and sampling type are entered in @Risk before the simulation is started. Latin Hypercube sampling is supported as a more effective method requiring fewer samples than traditional Monte Carlo sampling. @Risk calls Matlab/Simulink for each realisation with a new set of data samples. Simulink solves the model numerically and returns the result to @Risk so that statistics can be calculated. The different ecosystem blocks defined in Tensit are added to a common graphical library in Simulink and may be used in other modelling tasks. Hence it is easy to build complex models with interconnected blocks.

\subsection{A simple model in order to show the functionality of the code}

In this section a model which illustrates transport modelling with Tensit is presented. The model consists of two compartments (accumulators which handle nuclide decay) and two transfer function blocks specifying outflow of radionuclides from the compartments, Figure 1.

Five nuclides are simulated simultaneously, the nuclides used in the simulation are defined in the TENSIT block together with their respective half-lives and decay chains. There is an inflow to the system at a constant rate of one becquerel per year for each nuclide (if needed it is possible to simulate time dependent inflow, either as a function or interpolated data from file). In the current example, the flux is converted to moles per year by the Bq2Mole block and the influx to the compartment is hence given in moles. The two compartment blocks $A$ and $B$ have both a constant block attached which specifies the transfer coefficient, TC (outflux $=$ TC $\mathrm{x}$ amount of each nuclide in the accumulator). When simulating systems that changes with time, the transport coefficient may be given as a function (depending on time, amount of different nuclides in any compartment, speciation, inflow of nutrients etc.). Finally, compartment $A$ has a conversion block connected to its level out port so that the activity in the block is obtained. The activity in the block (or outflow or any other variable) may be written to file or used in any other downstream model in a more advanced case. 


\subsection{A more advanced model showing migration in a lake}

The conceptualisation of the lake model used in the safety assessment of a low and intermediate waste repository [4] is shown in Figure 2. This model is implemented in Tensit as a single block called Lake which may be reused in other systems. The lake model is hierarchically built up and is on the top level based on four compartments, each consisting of subcompartments (in the figure, the subcomparment representing the suspended matter is shown). The different compartments represents water, suspended matter, a top sediment in which the sediment may be resuspended and a deep sediment in which the nuclides are buried. The buried nuclides may appear as a source term in other models, for instance if the modelled lake is transferred to a mire (which is a natural succession of some small lakes at the studied site due to land rise).

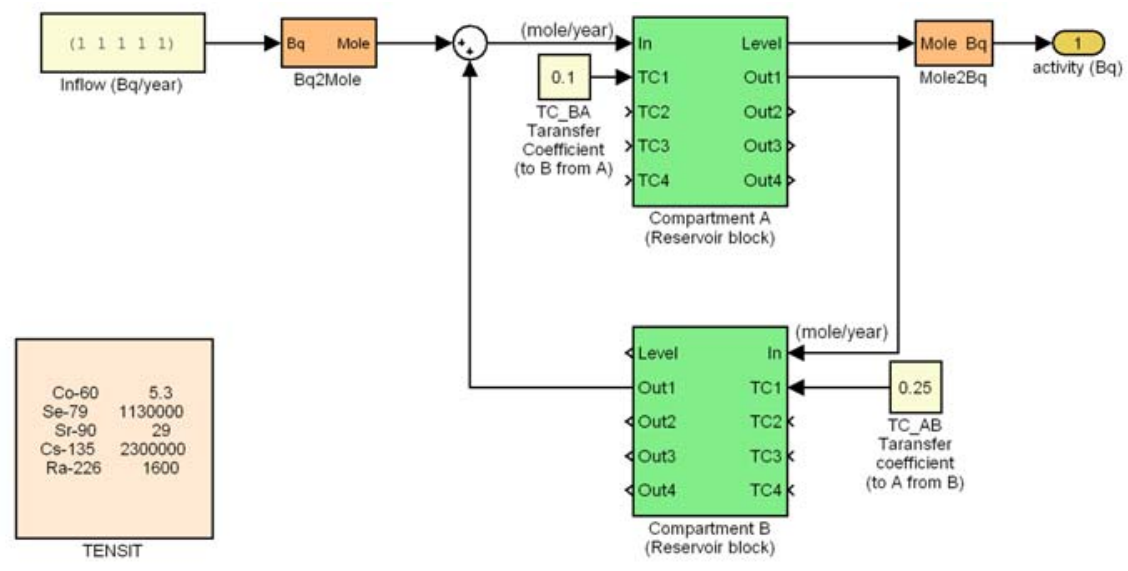

Figure 1. A model implemented in Tensit consisting of a number of separate reusable blocks. Five nuclides are simulated in a two compartment model. The inflow is $1 \mathrm{~Bq} / \mathrm{y}$ for each nuclide. The conversion blocks Bq2Mole and Mole2Bq convert activity to moles, and vice versa. The five nuclides, their decay behaviour and their half lives are specified in the TENSIT block.

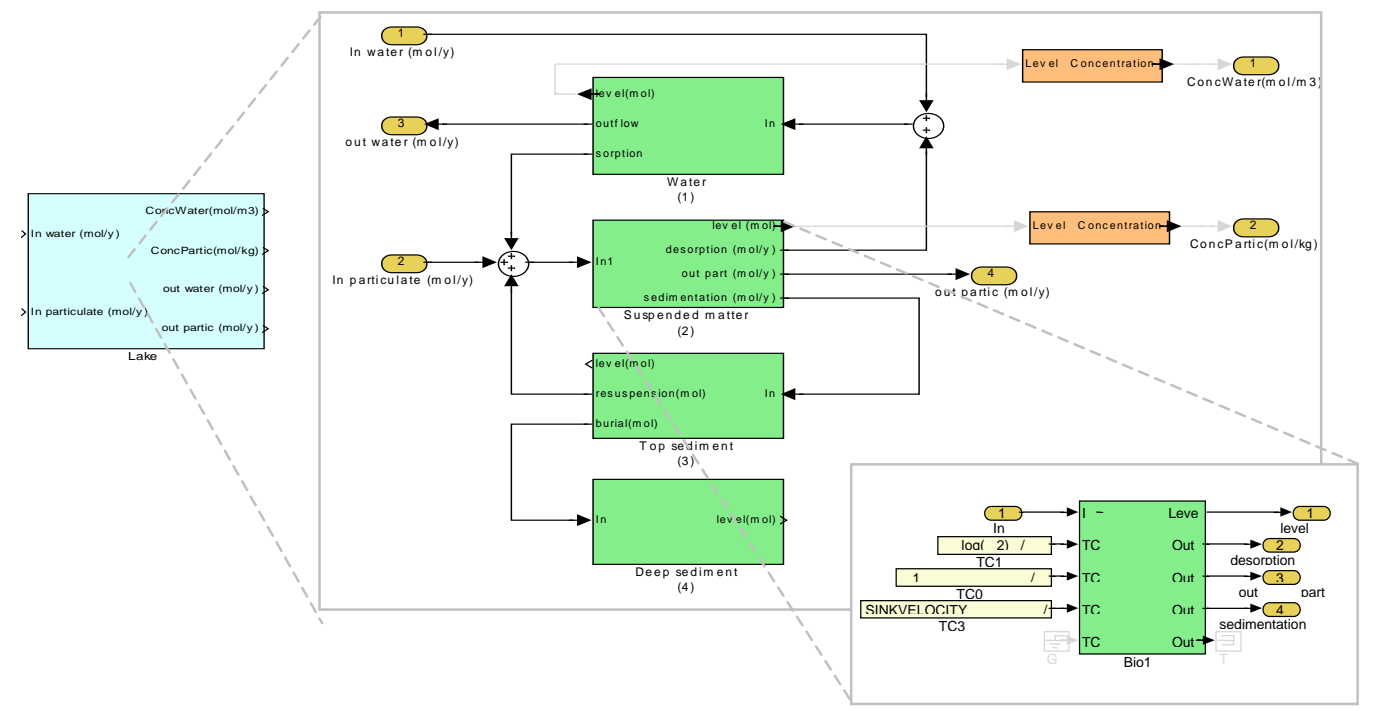

Figure 2. the Lake block consisting of four compartments with the smaller oval blocks representing in and out ports. The black lines represent transport between blocks and grey lines information of nuclide inventory in the compartments. The block representing the Suspended matter, is shown at a lower level which holds the actual integrating compartment and blocks defining outflow (using the transport function) from the block. 


\subsection{Connected ecosystems, the Landscape model}

As part of the safety assessment SR-Can an integrated landscape model consisting of interconnected biosphere objects have been developed, Figure 3 [5]. In this model radio nuclides are assumed to be released to the biosphere at several locations and being transported through a system of different biosphere objects (forest, costal area, lake and others). Different properties of the ecosystems at the site have been investigated [6] and field data obtained in the site investigation program have been refined to site specific input data for the blocks (the model uses site specific data for properties like geometrical information, retention times in the different lakes and mires etc.).

Hydrology models based on site specific data are used to define the locations of discharge points where (in which ecosystem blocks) the nuclides will enter the biosphere. Hydrology models are also used to define how the different ecosystem models are connected (through the surface water) and to define the discharge areas needed when defining the influx of water to biosphere objects like a lake and a mire needed to determine the concentration and the retention times.

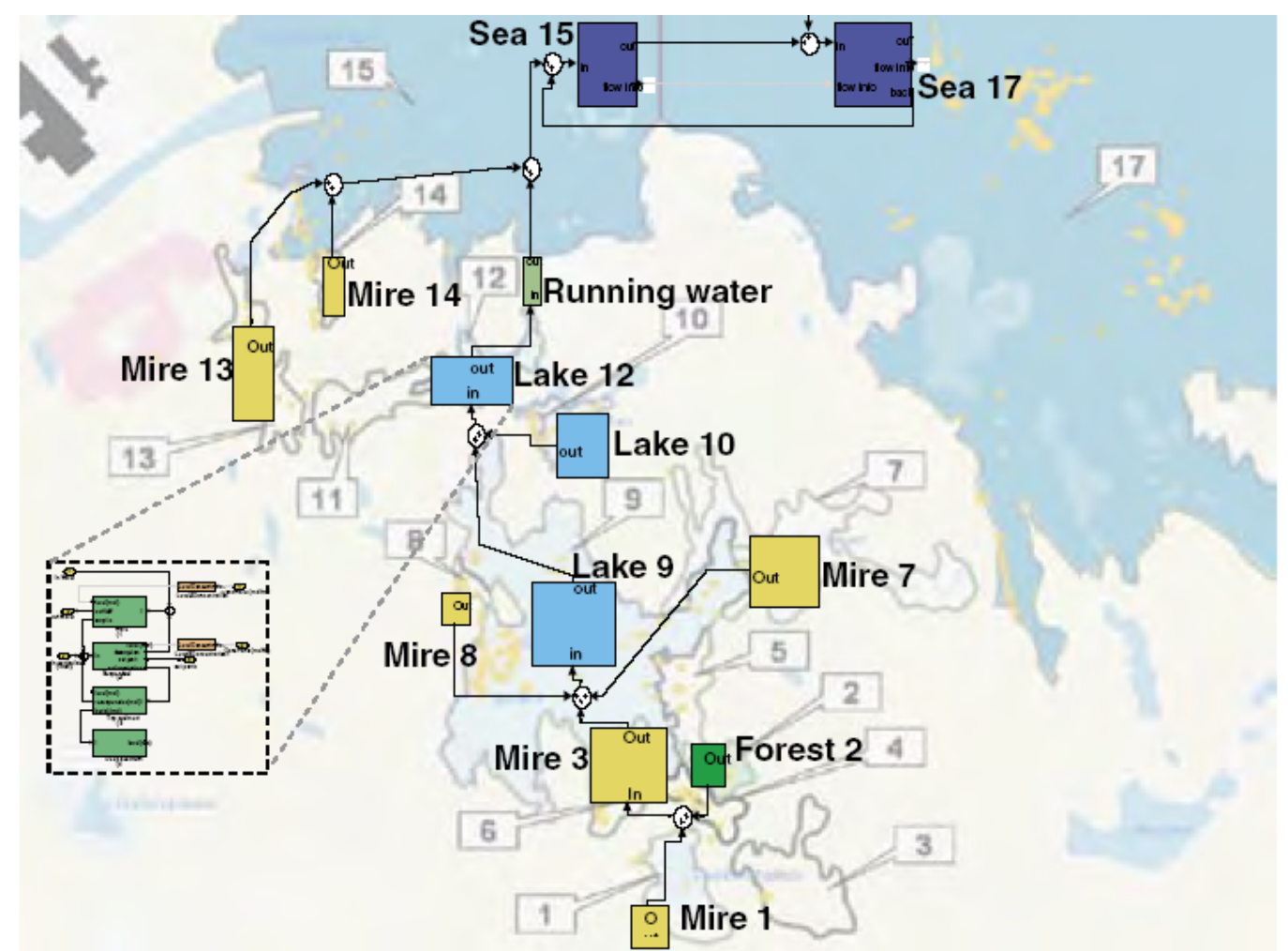

Figure 3. Landscape model consisting of a series of different interconnected ecosystem models. The underlying structure of lake 12 is presented in magnification. The numbers in the boxes represents biosphere objects characterised in the site investigation program. (From [7]).

\section{COMPARISONS WITH OTHER CODES}

To verify the accuracy of Tensit an implementation of the different biosphere modules used for the dose assessments in the safety assessment SR 97, [8] have been performed. Additional verifications using the AMBER [9] simulation code and against the international test case PSACOIN, Level 1B [8] have also been conducted. A detailed description of these studies and a collection of in and out data are available in [10]. 
In the SR97 safety assessment, an alternative code was used for the calculations and the purpose with this study is to investigate the agreement between the two both for deterministic as well as probabilistic simulations. The result from a probabilistic simulation for the SR97 Lake module (which is one of six different biosphere models simulated) is shown in Figure 4. Although the reference results are obtained after 200 realisations the Tensit implementation has been run 10000 realisations for increased accuracy and a better comparison between the codes. Only a few of the totally 43 nuclides falls just outside of the $95 \%$ confidence interval which all together indicates a good match and agreeing results.

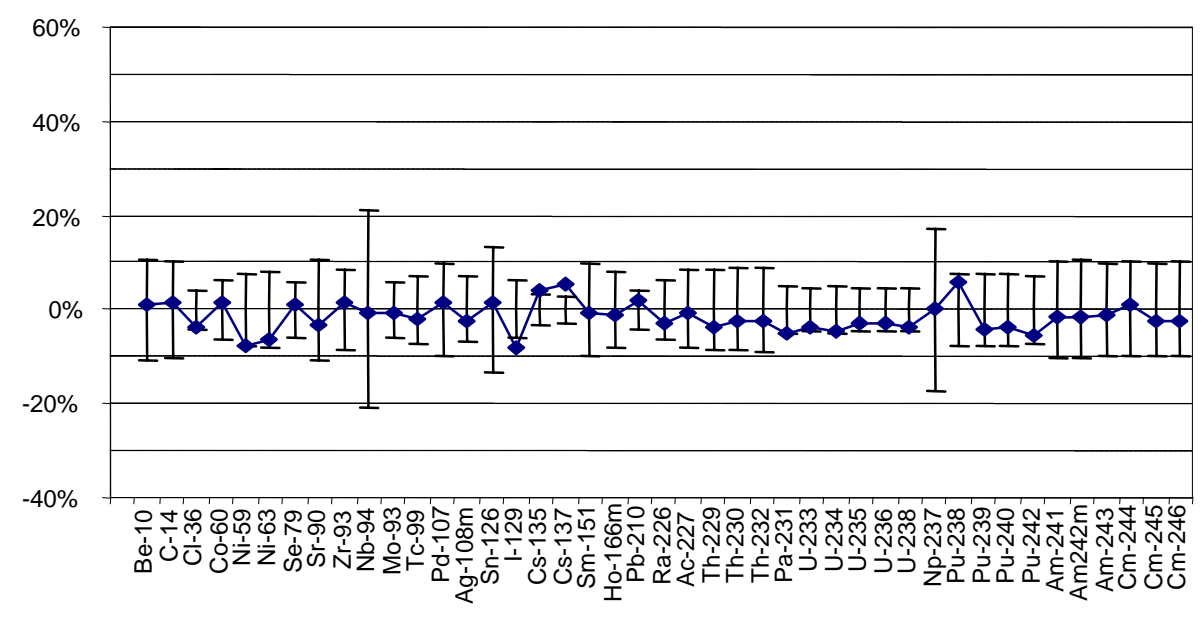

Figure 4. Comparison between Tensit and the reference code for an implementation of the SR 97 Lake model. The points plotted for each nuclide are the relative difference between the two results. The bars indicate the limits of a calculated $95 \%$ confidence interval for agreeing results.

In the comparison using the three test cases described in the Amber [9] a good agreement, between the two codes were shown [10].

The model used in the PSACOIN Level 1B exercise, [1], was implemented in Tensit. Using a specified test case, (a given dose model both deterministically and probabilistically) results from the eight participating groups have been compared and their results presented. Results from a Tensit implementation of that test together with the reported results are shown in Figure 5, the agreement between Tensit and the reported codes are excellent.

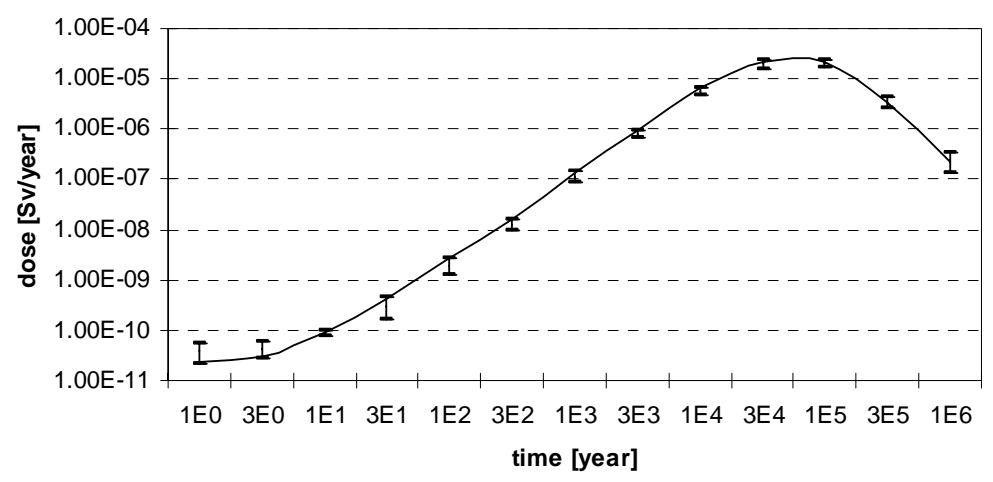

Figure 5. Dose rate from the ${ }^{235} \mathrm{U}$ chain. An interpolated line between data has been added. The vertical bars show the span of results reported from the eight participating groups. 


\section{DISCUSSION AND CONCLUSION}

In the present paper, the development of a probabilistic radionuclide migration simulation code based on pre-existing commercial software has been shown. The code shows a good agreement in comparisons with other codes used for migration calculations and is user friendly and well functioning. Future development will include a clearer graphical standard to better distinguish between transport and flow of information in the model. A new function block will be added that makes it easier to enter time dependent expressions in a single equation, instead of as now having to use at least a few blocks to build your time dependent expression. With this block built in algorithms to check mathematical consistency may also be included.

\section{References}

[1] R. A. Klos, J. E. Sinclair, C. Torres, U. Berström, D. A. Galson, PSACOIN Level 1B Intercomparison. (NEA; OECD, Paris, 1993).

[2] L. Kumblad, "Using ecosystem modelling techniques in exposure assessments of radionuclides - an overview.”, Ecorad, Aix an Provance 2004 , U. Kaustky.

[3] Mathworks, http://www.mathworks.com.

[4] S. Karlsson, U. Bergström, M. Meili, Models for dose assessments. Models adapted to the SFR-area, Sweden. SKB-TR-01-04, (SKB Swedish Nuclear Fuel and Waste Management, S-102 40 Stockholm, SWEDEN, 2001).

[5] U. Kautsky and L. Kumblad, SR-CAN - A safety assessment of a repository of spent nuclear fuel; canister performance and effects on the biosphere, Ecorad, Aix an Provance 2004.

[6] SKB, Preliminary site description Forsmark area - version 1.1. SKB R-04-15 (SKB Swedish Nuclear Fuel and Waste Management, S-102 40 Stockholm, SWEDEN, 2004).

[7] SKB, Interim Report of The Safety Assessment SR-Can, SKB-TR-04-11 (SKB Swedish Nuclear Fuel and Waste Management, S-102 40 Stockholm, SWEDEN, 2001).

[8] U. Bergström, S. Nordlinder and I. Aggeryd, Models for dose assessments. Modules for various biosphere types SKB-TR-99-14, (SKB Swedish Nuclear Fuel and Waste Management, S-102 40 Stockholm, SWEDEN, 1999).

[9] P. C. Robinson, J. S. S. Penfold, R. H. Little and R. C. Walke, AMBER 4.5 Verification: Summary, Document Reference Number: QRS-1059B-1, (Available August $18^{\text {th }} 2003$ at http://www.enviros.com)

[10] J. Jones, F. Vahlund and U. Kautsky, Tensit - a novel probabilistic simulation tool for safety assessments SKB-TR-04-07 (SKB Swedish Nuclear Fuel and Waste Management, S-102 40 Stockholm, SWEDEN, 2004). 